\title{
The Non-Degeneracy of Harmonic Structures on Planar Sierpinski Gaskets
}

\author{
Shiping $\mathrm{Cao}^{1}$ and Hua Qiu ${ }^{2, *}$ \\ ${ }^{1}$ Department of Mathematics, Cornell University, Ithaca 14853, USA \\ 2 Department of Mathematics, Nanjing University, Nanjing, Jiangsu 210093, China
}

Received 29 October 2019; Accepted (in revised version) 22 November 2019

Dedicated to Professor Weiyi Su on the occasion of her 80th birthday

\begin{abstract}
We present a direct and short proof of the non-degeneracy of the harmonic structures on the level- $n$ Sierpinski gaskets for any $n \geq 2$, which was conjectured by Hino in [1,2] and confirmed to be true by Tsougkas [8] very recently using Tutte's spring theorem.
\end{abstract}

Key Words: Fractal analysis, harmonic functions, fractal Laplacians, harmonic structures, Sierpinski gaskets.

AMS Subject Classifications: 28A80

\section{Introduction}

The theory of analysis on fractals, analogous to that on manifolds, has been being well developed. The pioneering work, developed by Kigami $[3,4]$, is the analytic construction of the Laplacians, for a class of finitely ramified fractals, named p.c.f. self-similar sets, including the Sierpinski gasket as a typical example, where Laplacians are defined as renormalized limits of graph Laplacians, playing the role of differential operators of second order on manifolds.

The harmonic functions on fractal domains may have some different nature from the classical ones as a consequence of the finitely ramified topology of the fractals. For example, the Hexagasket and the Vicsek set [7] consume degenerate harmonic structures so that nonconstant harmonic functions vanishing locally on small cells exist. It seems that such phenomenon always happens on those fractals containing nonjunction inner vertices.

Recently, Tsougkas [8] gives a proof on the non-degeneracy of harmonic structures on the level- $n$ Sierpinski Gasket $\mathcal{S G}_{n}, n \geq 2$, based on certain geometric graph theory, in

*Corresponding author. Email addresses: sc2873@cornell.edu (S. P. Cao), huaqiu@nju.edu.cn (H. Qiu) 

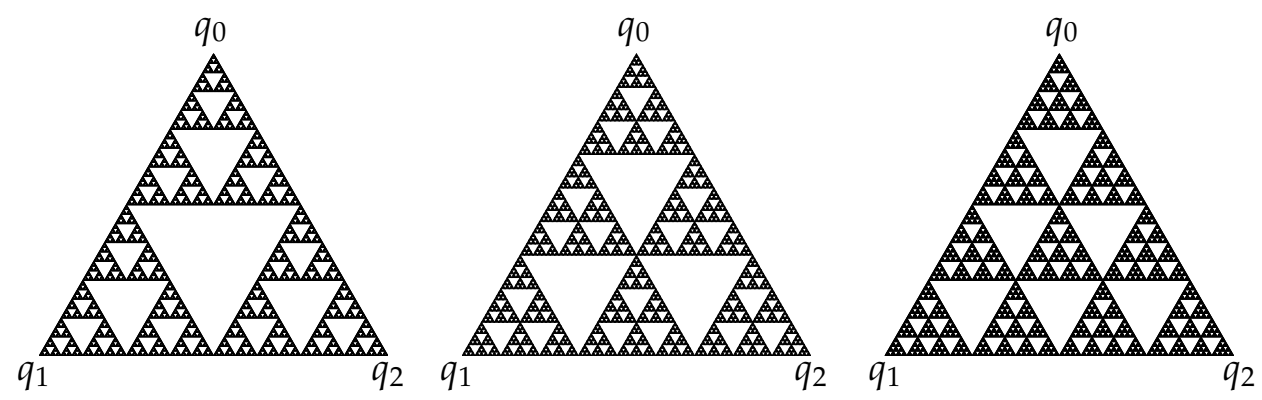

Figure 1: $\mathcal{S G}_{2}, \mathcal{S G}_{3}, \mathcal{S G}_{4}$.

which Tutte's spring theorem plays the key role, which was conjectured and numerically checked for the standard case for $n \leq 50$ by Hino [1,2].

In this note, we aim to provide an elementary proof for this theorem using only the basic facts of the discrete Laplacians on finite sets.

Theorem 1.1. For $n \geq 2$, each harmonic structure of $\mathcal{S G}_{n}$ is non-degenerate.

For convenience, we list some basic concepts and facts on the Laplacians on finite sets below. Readers can find any unexplained details in the book [5].

Definition 1.1. Let $V$ be a finite set. A symmetric linear operator (matrix) $H: l(V) \rightarrow$ $l(V)$ is called a Laplacian on $V$ if it satisfies

(1) $H$ is non-positive definite,

(2) $H u=0$ if and only if $u$ is a constant on $V$,

(3) $H_{p q} \geq 0$ for all $p \neq q$.

Denote $\mathcal{L} \mathcal{A}(V)$ the set of Laplacians on $V$.

Recall that $\mathcal{S G}_{n}$ is the unique nonempty compact subset of $\mathbb{R}^{2}$ satisfying

$$
\mathcal{S G}_{n}=\bigcup_{i=0}^{\frac{n^{2}+n-2}{2}} F_{i} \mathcal{S} \mathcal{G}_{n}
$$

with $F_{i}$ 's being contraction mappings defined as $F_{i}(z)=n^{-1} z+d_{n, i}$ with suitable $d_{n, i} \in$ $\mathbb{R}^{2}$. See Fig. 1 . The set $V_{0}$ consisting of the three vertices $q_{0}, q_{1}, q_{2}$ of the smallest triangle containing $\mathcal{S G}_{n}$ is called the boundary. In this note, we mainly discuss the Laplacians on

$$
V_{1}=\bigcup_{i=0}^{\frac{n^{2}+n-2}{2}} V_{0} .
$$



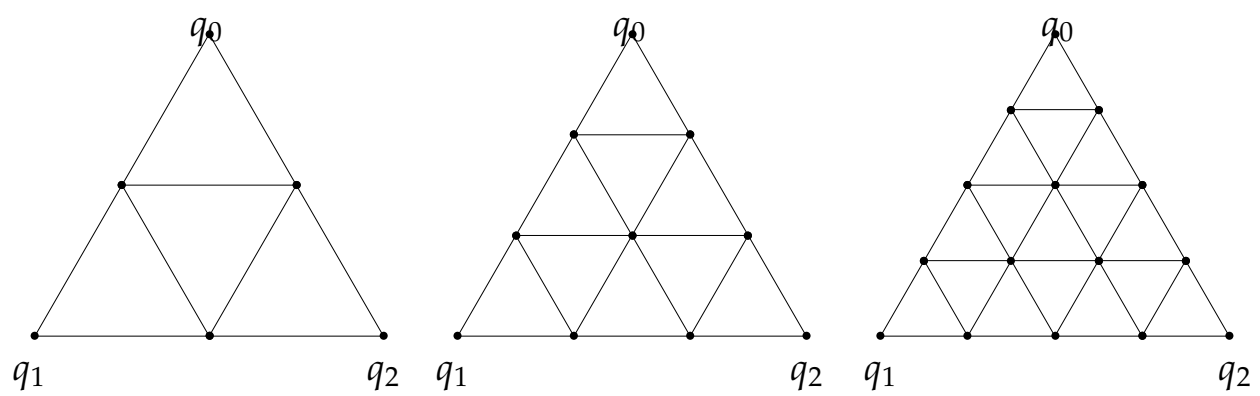

Figure 2: The level-1 graph approximation $G_{1}$ of $\mathcal{S G}_{2}, \mathcal{S G}_{3}, \mathcal{S G}_{4}$.

Let $D \in \mathcal{L} \mathcal{A}\left(V_{0}\right)$, and $\varepsilon_{0}$ be its associated Dirichlet form

$$
\varepsilon_{0}(u, v)=\sum_{p, q \in V_{0}} D_{p q}(u(p)-u(q))(v(p)-v(q)), \quad u, v \in l\left(V_{0}\right) .
$$

For $\mathbf{r}=\left(r_{0}, r_{1}, \cdots, \frac{r_{n^{2}+n-2}}{2}\right)$ with $r_{i}>0$, denote by $\mathcal{E}_{1}$ the induced Dirichlet form on $V_{1}$ with

$$
\mathcal{E}_{1}(u, v)=\sum_{i=0}^{\frac{n^{2}+n-2}{2}} r_{i}^{-1} \mathcal{E}_{0}\left(u \circ F_{i}, v \circ F_{i}\right), \quad u, v \in l\left(V_{1}\right) .
$$

We call the pair $(D, \mathbf{r})$ a harmonic structure if the restriction of $\varepsilon_{1}$ on $V_{0}$ equals $\varepsilon_{0}$. Once the pair $(D, \mathbf{r})$ is a harmonic structure, it could naturally induce a self-similar Dirichlet form on the fractal $\mathcal{S} \mathcal{G}_{n}$, see [5]. The Laplacian on $V_{1}$ associated with $\mathcal{E}_{1}$, denoted by $H_{1}$, can be expressed as

$$
H_{1}=\sum_{i=0}^{\frac{n^{2}+n-2}{2}} r_{i}^{-1} R_{i}^{t} D R_{i}
$$

where $R_{i}: l\left(V_{1}\right) \rightarrow l\left(V_{0}\right)$ is defined by $R_{i} v=v \circ F_{i}$.

By Theorem 3.2.11 in [5], it is easy to check that $\left(H_{1}\right)_{p q}>0$ if and only if $p, q \in$ $F_{i} V_{0}$ for some $0 \leq i \leq \frac{n^{2}+n-2}{2}$ due to the topology of $\mathcal{S G}_{n}$. We always denote $p \sim q$ if $\left(H_{1}\right)_{p q}>0$. The edge relation " $\sim$ " together with the vertex set $V_{1}$ gives the level-1 graph approximation $G_{1}$ of $\delta \mathcal{G}_{n}$. See Fig. 2 for $n=2,3,4$ cases.

Let $h$ be a harmonic function on $\mathcal{S} \mathcal{G}_{n}$ and $v=\left.h\right|_{V_{1}} \in l\left(V_{1}\right)$, then

$$
\left.\left(H_{1} v\right)\right|_{V_{1} \backslash V_{0}}=0 .
$$

Conversely, for any $v$ satisfying (1.1), it has a unique extension $h$ so that $h$ is harmonic on $\mathcal{S G}_{n}$. Thus we only need to study the solutions of (1.1). The following discrete maximum principle is important. See Proposition 2.1.7 in [5]. 
Proposition 1.1 (Maximum principle). Let $V$ be a finite set and $H \in \mathcal{L} \mathcal{A}(V)$. Let $U$ be $a$ proper subset of $V$. For $p \in V \backslash U$, write

$$
\begin{gathered}
U_{p}=\left\{q \in U: \text { There exist } p_{1}, p_{2}, \cdots, p_{m} \in V \backslash U \text { with } p_{1}=p H_{p_{i} p_{i+1}}>0\right. \\
\text { for } \left.i=1, \cdots, m-1 \text { and } H_{p_{m} q}>0\right\} .
\end{gathered}
$$

Then if $\left.(\mathrm{Hv})\right|_{V \backslash U}=0$, then for any $p \in V \backslash U$,

$$
\min _{q \in U_{p}} v(q) \leq v(p) \leq \max _{q \in U_{p}} v(q) .
$$

Moreover, $v(p)=\max _{q \in U_{p}} v(q)\left(\right.$ or $\left.v(p)=\min _{q \in U_{p}} v(q)\right)$ if and only if $v$ is constant on $U_{p}$.

\section{Main result}

Call a finite sequence of vertices $\left\{p_{1}, p_{2}, \cdots, p_{m}\right\} \subset V_{1}$ a chain if $p_{i} \sim p_{i+1}$ for $1 \leq i<m$ and $p_{i} \neq p_{j}$ for $i \neq j$. Before proving Theorem 1.1, we list some lemmas.

Lemma 2.1. Let $v \in l\left(V_{1}\right)$ satisfying (1.1), and assume $v$ is not a constant. If $p \in V_{1} \backslash V_{0}$ is a vertex with a neighbor $q \sim p$ such that $v(q) \neq v(p)$, then there exists a chain $\left\{p_{1}, p_{2}, \cdots, p_{m}\right\}$ with $p_{1}=p$ and $p_{m} \in V_{0}$ satisfying

$$
v\left(p_{1}\right)<v\left(p_{2}\right)<\cdots<v\left(p_{m}\right)
$$

Similarly, there is another chain satisfying $v\left(p_{1}\right)>v\left(p_{2}\right)>\cdots>v\left(p_{m}\right)$.

Proof. Let $p_{1}=p$. There must exist some $q \sim p_{1}$ such that $v\left(p_{1}\right)<v(q)$, since by (1.1),

$$
\left(H_{1} v\right)\left(p_{1}\right)=\sum_{p^{\prime} \sim p_{1}}\left(H_{1}\right)_{p_{1} p^{\prime}}\left(v\left(p^{\prime}\right)-v\left(p_{1}\right)\right)=0 .
$$

Set this $q$ as $p_{2}$. If $p_{2} \in V_{0}$, then we have already find the chain. Otherwise, repeat the above procedure until we find a $p_{m} \in V_{0}$. The process will stop after finite times of operations, since $V_{1}$ is a finite set.

Lemma 2.2. Let $A$ be a proper subset of $V_{1} \backslash V_{0}$ with at least two vertices. Write $\partial A=\{p \in$ $A \mid$ there exists $q \in V_{1} \backslash\left(A \cup V_{0}\right)$, such that $\left.\left(H_{1}\right)_{p q}>0\right\}$. Then \# $A \geq 2$.

Proof. If $\partial A=A$, then we immediately get the lemma. Otherwise, it is easy to see that $A \backslash \partial A$ and $V_{1} \backslash\left(A \cup V_{0}\right)$ are disconnected with each other, which also leads to \# $A \geq$ 2 as $\left(V_{1} \backslash V_{0}, \sim\right)$ is 2-connected. Here we call a graph 2-connected if it could not be disconnected by removing 1 vertex. 
Proof of Theorem 1.1. Let $v$ be the restriction of a nonconstant harmonic function $h$ on $V_{1}$. For $c \in \mathbb{R}$, define

$$
E(v, c)=\left\{p \in V_{1}: v(p)=c\right\}
$$

We only need to show that $F_{i} V_{0} \nsubseteq E(v, c)$ for any $0 \leq i \leq \frac{n^{2}+n-2}{2}$ and any $c \in \mathbb{R}$.

Assume $F_{i_{1}} V_{0} \subset E(v, c)$ for some $i_{1}$ and $c$. We may find another $i_{2}$ such that

$$
F_{i_{2}} V_{0} \subset E(v, c) \quad \text { and } \quad F_{i_{2}} V_{0} \bigcap F_{i_{1}} V_{0} \neq \varnothing .
$$

Also, another $i_{k}$ such that

$$
F_{i_{k}} V_{0} \subset E(v, c) \quad \text { and } \quad F_{i_{k}} V_{0} \bigcap\left(\bigcup_{l=1}^{k-1} F_{i_{l}} V_{0}\right) \neq \varnothing .
$$

Repeat the procedure until we can not find another such $i$, and set $A$ to be the union of these $F_{i_{k}} V_{0}$ 's. As a consequence of Lemma 2.2, there should be at least two vertices, denoted by $a_{1}, a_{2} \in A \backslash V_{0}$, such that for each $a_{i}$, there exists a neighbor $p \sim a_{i}$ with $v(p) \neq c$.

Let $C H=\left\{p_{1}, p_{2}, \cdots, p_{m}\right\} \subset A$ be a geodesic chain (with minimal number of edges, may not be unique) in $A$ connecting $p_{1}=a_{1}$ and $p_{m}=a_{2}$. See Fig. 3 for examples of such chains. The value of $v$ is always $c$ along this chain. On the other hand, for each $i=1,2$, by using Lemma 2.1, we could connect $a_{i}$ to the boundary $V_{0}$ by two different chains $C H^{S, i}$ and $C H^{L, i}$, denoted as

$$
\begin{array}{ll}
C H^{S, i}=\left\{p_{k}^{S, i}\right\}_{k=1}^{m_{S, i}} & \text { with } p_{1}^{S, i}=a_{i} \text { and } p_{m_{S, i}^{S, i}} \in V_{0}, \\
C H^{L, i}=\left\{p_{k}^{L, i}\right\}_{k=1}^{m_{L, i}} & \text { with } p_{1}^{L, i}=a_{i} \text { and } p_{m_{L, i}, i}^{L} \in V_{0},
\end{array}
$$

satisfying that $p_{k}^{S, i}<c, \forall 2 \leq k \leq m_{S, i}$ and $p_{k}^{L, i}>c, \forall 2 \leq k \leq m_{L, i}$.

Take a vertex $p \in A \backslash C H$ such that $p \sim p_{i_{1}}, p \sim p_{i_{2}}$ for two different $p_{i_{1}}, p_{i_{2}} \in C H$. Note that $v(p)=c$. It is not possible for $p \in V_{0}$, otherwise it will contradict to the fact
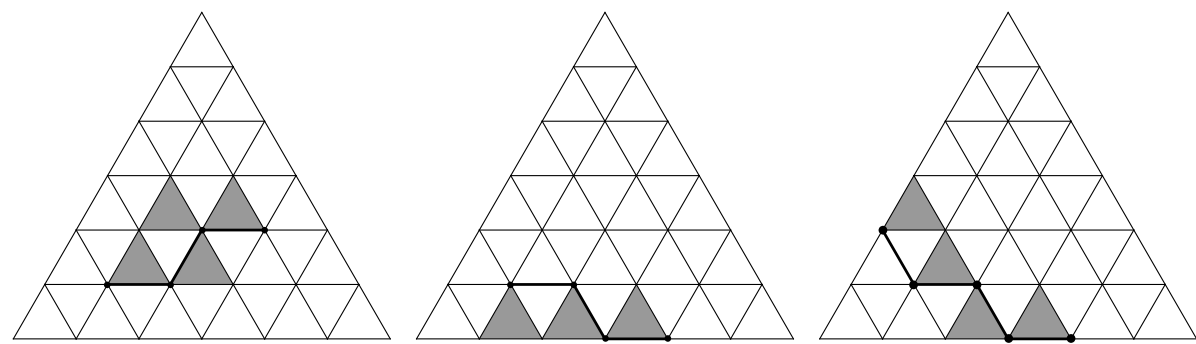

Figure 3: Examples of possible cases for $\mathrm{CH}$. 
that there exist five chains as shown above, since $C H^{S, i}$ and $C H^{L, j}$ never cross when $i \neq j$. Now as in Proposition 1.1, we take $U$ to be the union of $V_{0}$ and above five chains, and $V$ to be $V_{1}$. Noticing that $p \in V \backslash U$, we have

$$
\text { either } U_{p} \subset C H \cup C H^{S, 1} \cup C H^{S, 2} \quad \text { or } \quad U_{p} \subset C H \cup C H^{L, 1} \cup C H^{L, 2} \text {. }
$$

Moreover, since $\mathrm{CH}$ is a geodesic chain, it could not encircle a nonempty subset of $V_{1} \backslash V_{0}$, and thus there must exist a chain in $V_{1} \backslash C H$ which connects $p$ to some $q_{i} \in V_{0}$. So we have

$$
U_{p} \backslash C H \neq \varnothing .
$$

By (2.1), (2.2) and the definition of $C H^{S, i}$ and $C H^{L, i}$, we have either $\min _{q \in U_{p}} v(q)<$ $c=\max _{q \in U_{p}} v(q)$ or $\min _{q \in U_{p}} v(q)=c<\max _{q \in U_{p}} v(q)$. Thus we get $v(p) \neq c$ by using the maximum principle as stated in Proposition 1.1. However, this contradicts to the fact that $p \in A$.

As we know, harmonic functions on $\mathcal{S} \mathcal{G}_{n}$ are of 3-dimensional, and could be uniquely determined by their boundary values on $V_{0}$. More precisely, there exist harmonic extension matrices $A_{i}, 0 \leq i \leq \frac{n^{2}+n-2}{2}$, so that

$$
\left.h\right|_{F_{i} V_{0}}=\left.A_{i} h\right|_{V_{0}}
$$

holds for any harmonic function $h$ on $\mathcal{S G}_{n}$. We could restate Theorem 1.1 as following.

Corollary 2.1. All the harmonic extension matrices $A_{i}$ of $\mathcal{S G}_{n}$ are nondegenerate.

Proof. Let $h$ be a harmonic function and let $v=\left.h\right|_{V_{1}}$. If $\left.v\right|_{V_{0}}=c \neq 0$, then $\left.v\right|_{F_{i} V_{0}}=c$. If $\left.v\right|_{V_{0}}$ is not a constant, then by Theorem 1.1, $\left.v\right|_{F_{i} V_{0}}$ is also not a constant. These show that $\left.A_{i} v\right|_{V_{0}} \neq 0$ if $\left.v\right|_{V_{0}} \neq 0$.

\section{Acknowledgements}

The research of the second author was supported by the Nature Science Foundation of China, Grant No. 12071213.

\section{References}

[1] M. Hino, Some properties of energy measures on Sierpinski gasket type fractals, J. Fractal Geom., 3 (2016), 245-263.

[2] M. Hino, Energy measures and indices of Dirichlet forms, with applications to derivatives on some fractals, Proc. Lond. Math. Soc., 100.1 (2009), 269-302.

[3] J. Kigami, A harmonic calculus on the Sierpinski spaces, Japan. J. Appl. Math., 6 (1989), 259-290.

[4] J. Kigami, Harmonic calculus on p.c.f. self-similar sets, Trans. Amer. Math. Soc., 335 (1993), 721-755. 
[5] J. Kigami, Analysis on Fractals, Cambridge University Press, 2001.

[6] R. S. Strichartz, Some properties of Laplacians on fractals, J. Funct. Anal., 164 (1999), 181208.

[7] R. S. Strichartz, Differential Equations on Fractals: A Tutorial, Princeton University Press, 2006.

[8] K. Tsougkas, Non-degeneracy of the harmonic structure on Sierpinski Gaskets, J. Fractal Geom., 6(2) (2019), 143-156. 